\title{
Case 2 / 2019 - Complete Atrioventricular Septal Defect, with Down Syndrome, without Pulmonary Hypertension and Natural History at 33 Years of Age
}

\author{
Edmar Atik, ${ }^{\oplus}$ Alessandra Costa Barreto, Maria Angélica Binotto \\ Instituto do Coração do Hospital das Clínicas da Faculdade de Medicina da Universidade de São Paulo, São Paulo, SP - Brazil
}

\section{Clinical data}

The patient has developed no symptoms since birth, when heart disease was diagnosed by the presence of heart murmur in the presence of physical elements of Down syndrome. The patient is currently able to walk approximately 30 minutes daily without showing signs of fatigue and accompanies other individuals normally. The patient uses enalapril and levothyroxine for hypothyroidism.

Physical examination: good overall status, eupneic, acyanotic, normal pulses in the 4 limbs. Weight: $41 \mathrm{~kg}$, Height: $131 \mathrm{~cm}, \mathrm{MBP}$ in the right upper limb: $110 \times 60 \mathrm{mmHg}$, HR: 93 bpm, Sat $\mathrm{O}_{2}$ : 97\%.

Precordium: non-palpable ictus cordis, without systolic impulses. Accentuated and constant split second heart sound. Moderate intensity holosystolic murmur at the left lower sternal border and ejection murmur at the high border. Non-palpable liver and lungs clear to auscultation.

\section{Complementary examinations}

Electrocardiogram: Sinus rhythm, with no signs of cavitary overload and/or conduction disturbances, with a narrow QRS of $0.87 \mathrm{~ms}\left(\mathrm{AQRS}=-10^{\circ}\right)$, positive T-wave at $\mathrm{V} 1\left(\mathrm{AT}=0^{\circ}\right)$, and normal P-wave (AP $=30^{\circ}$ ) (Figure 1).

Chest X-ray: Slightly enlarged cardiac silhouette due to double atrium arch and left ventricular arch $(\mathrm{CTI}=0.50)$. Normal pulmonary vascular network (Figure 1).

Echocardiogram: Atrioventricular and ventriculoarterial connections concordant with ostium primum-type atrial septal defect measuring $10 \mathrm{~mm}$ in diameter and inflow tract ventricular septal defect measuring $15 \mathrm{~mm}$, with an effective 7-mm orifice by interposition of valve tissue, with interventricular pressure gradient of $78 \mathrm{mmHg}$. Single atrioventricular valve with two orifices, of which the right one measured $23 \mathrm{~mm}$ and the left, $30 \mathrm{~mm}$. There was left valve regurgitation caused by cleft, resulting in left atrial

\section{Keywords}

Heart Defects, Congenital; Complete atrioventricular defect Clinical/surgery. Down syndrome; Radiography, Thoracic/methods; Echocardography/methods.

Mailing Address: Edmar Atik •

Private office. Rua Dona Adma Jafet, 74, conj.73, Bela Vista. Postal Code 01308-050, São Paulo, SP - Brazil

E-mail: conatik@incor.usp.br

DOI: $10.5935 / a b c .20190024$ increase. Regurgitation on the right was discreet. The right ventricular systolic pressure was $39 \mathrm{mmHg}$. The cavities had the following dimensions: $\mathrm{RV}=16, \mathrm{LV}=54, \mathrm{LA}=45$, Ao $=25$, PAs $=17 \mathrm{~mm}$ (Figure 2).

Clinical diagnosis: Complete atrioventricular septal defect, with discrete repercussion caused by left atrioventricular valve regurgitation and small manifestation due to left-to-right blood shunting in atrioventricular position with Down's syndrome, in the presence of the natural history of the disease up to adulthood.

Clinical reasoning: There were clinical elements that offered a diagnostic indication of acyanogenic congenital heart disease with a slight clinical repercussion, such as atrial septal defect (ASD) and ventricular septal defect (VSD), in the presence of characteristic auscultation. The electrocardiogram did not show any cavitary overload and the chest X-ray showed a slight preponderant enlargement of the left cavities. The echocardiogram highlighted the diagnostic elements of the defect.

Differential diagnosis: Other acyanogenic cardiopathies such as ASD and / or VSD should be remembered in this context. Regurgitation of the valve on the left could also raise doubts regarding the diagnosis of the partial atrioventricular septal defect.

Clinical conduct: Considering the pulmonary and systemic flow balance over time, with no signs of hypoxemia and / or heart failure and in the presence of good physical tolerance, an expectant conduct was considered.

Comments: It is known that the complete atrioventricular septal defect has an unfavorable evolution, with signs of heart failure after the reduction of the pulmonary vascular resistance at a few days of life, in addition to progressive pulmonary vascular disease from a few months until the end of the first year of life. Thus, the need for early surgical intervention, in the first months of life, when there is a good long-term postoperative evolution. ${ }^{1}$

It can be affirmed that cases with an atrioventricular septal defect with discrete repercussion, of which evolution remains asymptomatic until adult life, are rarely seen. In this condition, the patients do not require early surgical intervention. Therefore, it is important to emphasize that these patients need a stringent and thorough evaluation, aiming to determine the best conduct for the infant, whether expectant or a surgical intervention. However, it is a difficult decision to make, since we have not found in the literature similar reports to the case described herein. 


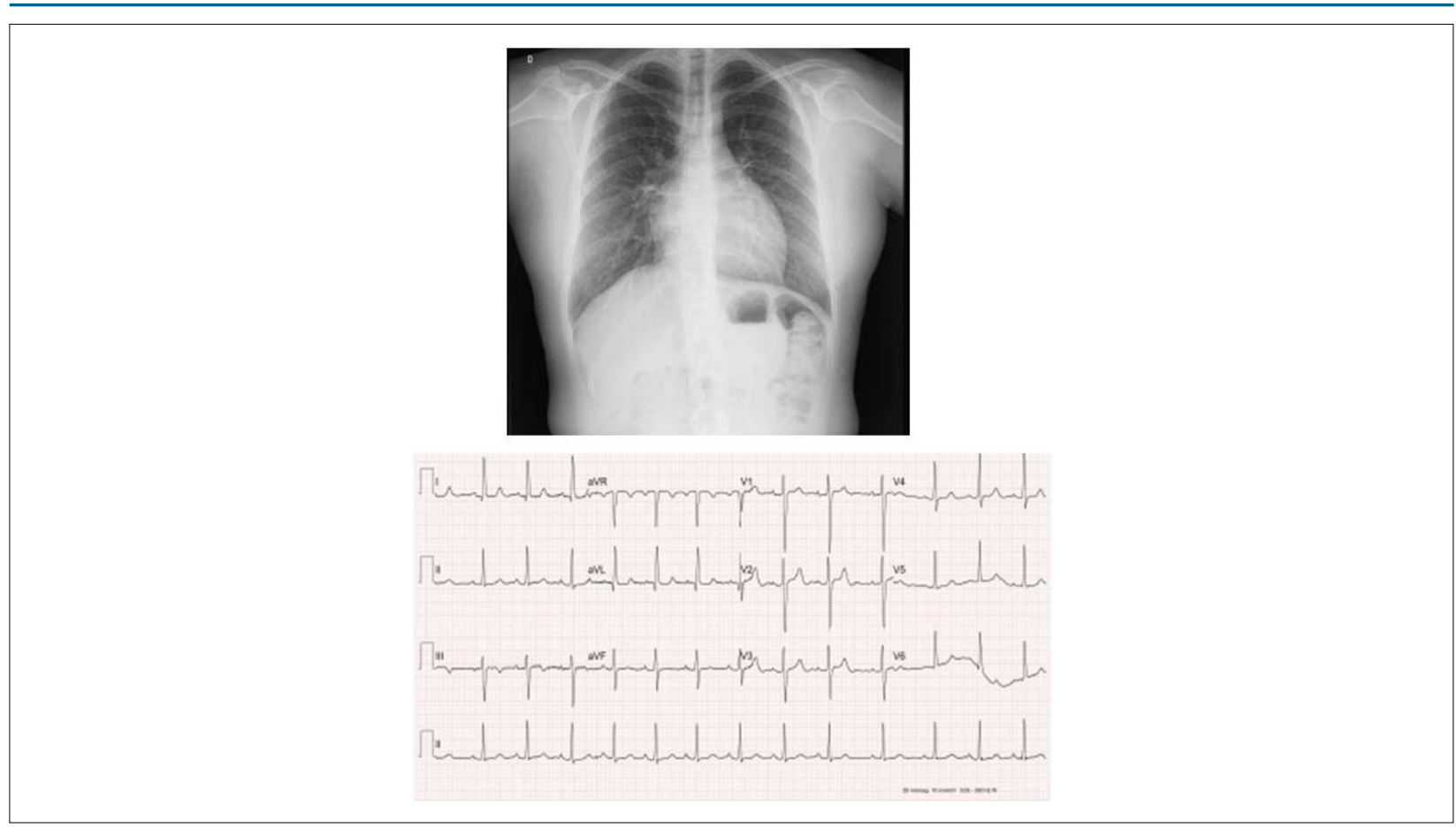

Figure 1 - Chest radiography showing the slightly enlarged cardiac silhouette with normal pulmonary vascular network, pulmonary and systemic flow balance. Electrocardiogram shows normal without overload or conduction disturbances.
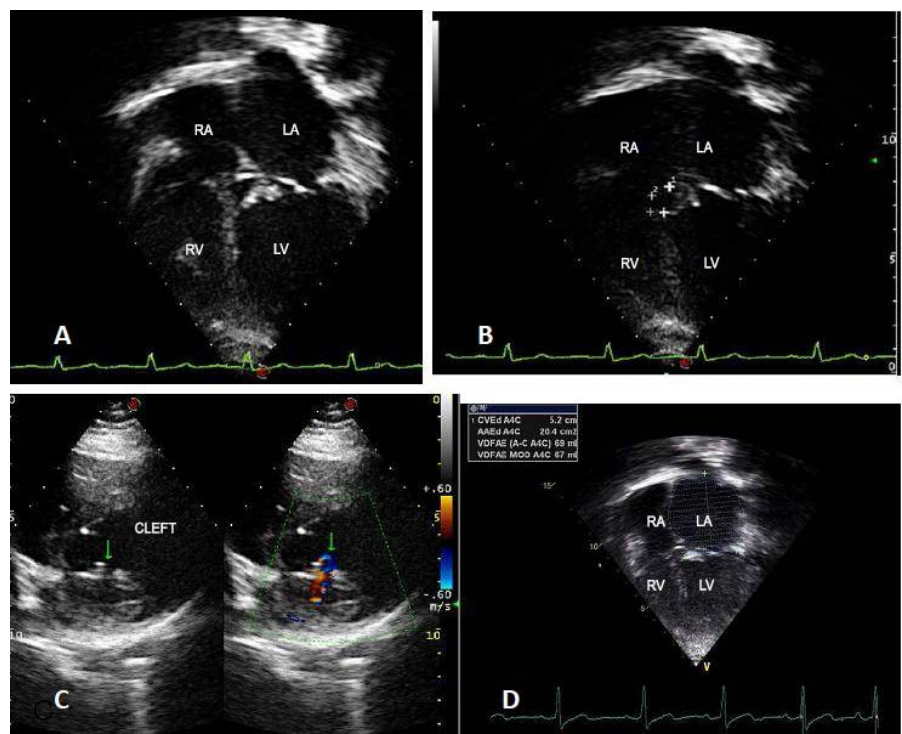

Figure 2 - Echocardiogram showing in the 4-chamber apical plane, the increase in the left atrium due to the discrete regurgitation of the left atrioventricular valve caused by a cleft in $\mathrm{C}$. The other cavities are normal due to closure of the inflow tract interventricular septal defect and the interatrial septal defect by valvular tissues, in $A, B$ and $D$.

\section{Reference}

1. Ginde S, Lam J, Hill GD, Cohen S, Woods RK, Mitchell ME, et al. Long-term outcomes after surgical repair of complete atrioventricular septal defect. J Thorac Cardiovasc Surg. 2015;150(2):369-74 